\title{
Estrategia de marketing digital para el posicionamiento en el mercado en el ramo de panaderías
}

Digital marketing strategy for market positioning in the bakery bouquet

\begin{tabular}{|rrr|}
\hline Marianny Aponte & Yndhira Beiza & Eliana Durán \\
\hline mariannyaponte88@hotmail.com & yndhirabeiza@gmail.com & eliana_duran2528@hotmail.com \\
ORCID: 0000-0001-5548-4071 & ORCID: 0000-0002-6166-2732 & ORCID: 0000-0003-3619-1745 \\
Universidad de Carabobo, Venezuela & Universidad de Carabobo, Venezuela & Universidad de Carabobo, Venezuela \\
\hline
\end{tabular}

Artículo recibido en enero 2020 / Arbitrado en febrero 2020 / Publicado en mayo 2020

RESUMEN | El objetivo de la investigación fue proponer el marketing digital como estrategia que permita el posicionamiento en el mercado de una empresa de Panadería. Metodológicamente se apoyó bajo la modalidad de proyecto factible, investigación descriptiva con diseño de campo. La población y muestra estuvo conformada por diez (10) empleados de la empresa y diez (10) clientes elegidos al azar, a través de dos cuestionarios con escalamiento de Likert. Se puede concluir que se evidencian deficiencia en las estrategias de marketing de la empresa, no existe personal capacitado para el uso de medios sociales, como estrategia de mercadeo digital, lo cual es propicio para la elaboración de la propuesta para posicionarse en el mercado local y regional.

Palabras clave:

Captación de clientes, Estrategias, Marketing digital, Mercado

\begin{abstract}
The objective of the research was to propose digital marketing as a strategy that allows a bakery company to position itself in the market. Methodologically, it was supported under the modality of feasible project, descriptive research with field design. The population and sample consisted of ten (10) company employees and ten (10) clients chosen at random, through two questionnaires with Likert scaling. It can be concluded that there are deficiencies in the company's marketing strategies there is no trained personnel to use social media as a digital marketing strategy, which is conducive to the development of the proposal to position itself in the local market and regional.
\end{abstract}

Keywords: Customer acquisition, Strategies, Digital Marketing, Market, Positioning 


\section{INTRODUCCIÓN}

Actualmente muchas de las empresas a nivel mundial, indistintamente de su tamaño, invierten una parte de su presupuesto en estrategias y planificaciones de mercadeo, que les permita crecer, y posicionarse como marca ante el mercado, que hoy en día se vuelve más exigente, gracias a la economía inestable, los procesos tecnológicos, y a la innovación en la calidad del servicio, que el cliente espera al momento de adquirir un producto.

En tal sentido, el marketing se puede decir que es el conjunto de actividades dirigidas a la creación de los mercados o a la consecución de participaciones en mercados ya existentes, satisfaciendo las necesidades de los consumidores con determinados productos o servicios, obteniendo un beneficio para la empresa.

Es importante destacar que en la actualidad las organizaciones se enfrentan a un mercado cada vez más competitivo y cambiante, en donde no tener una visión a futuro de lo que se quiere lograr podría ser un obstáculo para alcanzar el éxito. Las empresas se ven en la necesidad de tomar decisiones que les permitan adaptarse a dichos cambios y desarrollar a su vez la capacidad de competir en el mercado.

De tal modo, las Tecnologías de la Información y Comunicación (TC), constituyen una herramienta esencial para que las empresas mejoren su eficiencia y la calidad de sus productos y servicios, así como para fomentar su inclusión en el mercado. La utilización de las TIC'S, contribuyen de manera importante al proceso de innovación empresarial, debido a que mejoran el acceso y difusión de la información, obteniendo así, mayor eficacia en la presentación, sintetización y procesamiento de la información, lo que ha impulsado a varios países del mundo a tener este tipo de tecnologías como también a producirlas. En relación, a cómo ha cambiado el consumidor en esta era digital y su utilización de las diferentes redes, Vargas (2015), señala que:

Ahora vivimos conectados a diferentes dispositivos electrónicos que hacen nuestra vida y comunicación más sencilla. Celulares, Smart Phones (o teléfonos móviles multifuncionales), reproductores de audio y video, consolas de videojuegos, computadores son sólo algunos de ellos. Hemos cambiado nuestros hábitos y maneras de relacionarnos con las personas. Facebook se ha convertido en el parque del barrio en donde salimos a conocer y hacer amigos, Flickr se ha convertido en el museo virtual de fotografía más importante del mundo; YouTube, se está convirtiendo en la videoteca de la humanidad; eBay es la tienda en donde podemos comprar y vender de todo desde la comodidad de nuestra casa. Internet nos está imbuyendo en una nueva manera de relacionarnos con el mundo y con sus cosas (p.21).

Para conseguir el éxito en la actualidad, es indispensable contar con una dirección 
estratégica, es decir, una empresa debe desarrollar al máximo todo su potencial a través de mecanismos que le permitan obtener beneficios y sobrevivir por largo tiempo en un mercado cada vez más grande y competitivo. Esta corriente de intercambios económicos, obliga irremediablemente a las empresas prestadoras de algún servicio, a abrirse paso creativamente en este tan dinámico y al mismo tiempo, competitivo mundo. En efecto, no es lo común que una empresa exista sola en el mercado, sino, al contrario, lo usual es verla forzada a competir con otras iguales a ella que traten de satisfacer las mismas necesidades de un mismo grupo de consumidores.

En el mundo, así como en Venezuela las pequeñas y medianas empresas contribuyen en gran medida al desarrollo del país. Estas soportan las operaciones de las grandes corporaciones, ofrecen mayor número de empleos y permiten desarrollar el espíritu empresarial. Para crear diferenciación las empresas necesitan identificar las necesidades de su mercado meta, así como la mejor forma de satisfacerlas; esto mediante planes de mercadotécnica generados por el trabajo integrado de las diferentes áreas de la organización, financieros, humanos, tecnológicos. Además, de la identificación de necesidades el plan debe encaminar el rumbo de las actividades diarias de la organización con una mejor estructura organizacional para utilizar los recursos. De acuerdo a lo planteado, se requiere dentro del proceso de mercado aplicar estrategias de promoción de los productos.
Hoy en día, todas las organizaciones enfocan sus principales esfuerzos en el desarrollo de las estrategias de mercadeo que, enlazadas con las estrategias empresariales le permiten participar de manera activa y competitiva dentro de mercados internos e internacionales logrando no sólo posicionar una marca o productos sino establecerse en la mente de clientes y consumidores como su máxima en la decisión de compra.

Unos de estos casos es la Panadería y Pastelería Dorada, C.A, la cual se dedica, a la elaboración de pan, tortas y repostería, sin embargo, presenta en la actualidad un problema de identificación por parte del público consumidor, ya que la mayoría de los clientes se sorprenden al saber de la existencia de la misma y la variedad de productos con la que cuenta, debido a que no cuenta con un plan publicitario para su promoción y conocimiento, limitando el alcance las metas de productividad $y$ posicionamiento planteadas. De allí que como producto de esta investigación, se proponga un plan de marketing digital como estrategia que permita el posicionamiento en el mercado de la Panadería y Pastelería Dorada, C.A.,

\section{MATERIALES Y MÉTODO}

Ésta investigación se desarrolló bajo la modalidad de proyecto factible, ya que la misma tiene como objetivo general Proponer el marketing digital como estrategia que permita el posicionamiento en el mercado de la Panadería y Pastelería Dorada, C.A. En 
cuanto al nivel de profundidad de la investigación, fue tipo descriptiva debido, a que durante su realización, se hizo una narrativa exponiendo la problemática planteada, describiendo algunas características esenciales de la realidad estudiada, permitiendo así obtener una acertada interpretación de lo que ocurre en el objeto de estudio.

El diseño de investigación, fue de campo, por tanto las informaciones son recolectadas directamente y de fuentes primarias, en este caso el objeto de estudio la Panadería y Pastelería Dorada, C.A. La población y muestra, en este caso población estuvo conformada por 20 individuos, siendo diez (10) empleados del Área de Administración de la Panadería y Pastelería Dorada y los otros diez (10) individuos, clientes de la panadería tomados al azar.

La técnica y recolección de datos para dar respuesta a los objetivos planteados en esta investigación fue el cuestionario (se aplicaron dos (02) cuestionarios; uno dirigido a los clientes compuesto por doce (12) ítems y otro a los empleados de la panadería objeto de estudio conformado por diecisiete (17) ítems, con una escala de Likert), cuyos resultados se concentran en la matriz DOFA, presentada en este artículo y no se reproducen textualmente por razones de extensión del documento.

\section{RESULTADOS Y DISCUSIÓN}

Las proposiciones teóricas dedicadas a explicar cómo crear, ganar y dominar los mercados a través de la evolución de las estrategias de marketing empresarial han sido desarrolladas por diversos autores, como Kotler (2002), prolífico autor de libros sobre marketing desde la década de los 80's. El tema central de sus proposiciones con respecto a este tópico se basa en el abandono de las ideas, por parte de las empresas, de lo que él mismo denomina como marketing obsoleto, el viejo marketing, y de equiparar este mercadeo con las ventas, no sólo produciendo bienes, sino adaptándolos a lo que desea el mercado.

No obstante, aunque se han realizado exhaustivos estudios sobre marketing y su integración al sistema estratégico de las empresas hasta formar parte de su columna vertebral como organizaciones que compiten en el mercado para ofrecer valor a sus clientes, aun así, estudios exploratorios recientes efectuados por Peinado y Prado (2013), exponen la necesidad:

Sobre todo a nivel de pequeñas y
medianas empresas, de incorporar
un plan de marketing a sus
estrategias de mercado para
aumentar su productividad y
competitividad, la implementación
de herramientas se considera el
móvil para impulsar la imagen, la
razón de ser de la empresa, sus
productos y cada uno de los
servicios que estos entes pudiesen
prestar (p.35).

En este mismo orden de ideas, y tomando en cuenta lo anterior, se plantea la siguiente definición según Hughes (2007:48): 
"La promoción es el conjunto de actividades, técnicas y métodos que se utilizan para lograr objetivos específicos, como informar, persuadir o recordar al público objetivo, acerca de los productos $y / 0$ servicios que se comercializan". En este caso la promoción es una herramienta fundamental para dar a conocer las bondades y beneficios de los productos, las empresas que no utilizan esta herramienta desmejoran su presencia en el mercado y alteran las condiciones económicas y operativas de la empresa.

En este sentido se debe resaltar que las Tics, facilitan un sin fin de herramientas fundamentales para la promoción, manejo y desarrollo de los marketing es por ello, que primero se analizaron la teoría de Información y Comunicación y luego las Tecnologías de Información y Comunicación (TIC'S), que llevará a entender cómo ha evolucionado las mismas, y hoy en día se conocen las redes sociales, y su adaptación al marketing digital.

Las tecnologías de información y comunicación constituyen hoy en día los nuevos canales de comunicación, que afecta todo el entorno y afecta de alguna manera la vida, éstas nuevas tecnologías forman cada vez más parte de las culturas, por lo tanto es importante tener los conocimientos apropiados para darles el uso adecuado y obtener así, los mejores beneficios de intercambio y obtención de información.

Las TIC, se ha convertido en un factor dominante tanto de las organizaciones como en la vida personal. Según Koontz y Weihrich (1998), "es la suma total de conocimientos sobre la forma de hacer las cosas, incluyendo inventos, técnicas y el vasto acervo de conocimientos organizados"; mientras Gaynor (1999), establece su denominación, "en función de un conjunto de medios creados por personas para facilitar el esfuerzo humano". Valdes (2000), la define como "un método o procedimiento para efectuar algo".

Todos esos puntos de vista, efectuados por diversos autores, coinciden que la tecnología, es un conjunto de nociones o ideas orientadas al desarrollo de un sector, el cual incluye para su desenvolvimiento el uso de procedimientos, herramientas, instrucciones y conocimientos científicos, que auxilian el perfeccionamiento para la satisfacción de las necesidades del consumidor. La concepción moderna de las tecnologías de información y comunicación afirma Gil (2002), que:

Comprende aplicaciones, sistemas, herramientas, técnicas y metodologías asociadas a la digitalización de señales analógicas, sonidos, textos e imágenes, manejables en tiempo real. Asimismo se relaciona con equipos de computación, software, telecomunicaciones, redes y bases de datos, lo que permite destacar que la evolución del proceso humano de recibir información y comunicarse, está estrechamente relacionada con la evolución tecnológica, pues trae consigo transformaciones a nivel comercial, educativo, cultural, social y económico, por su carácter global, accesible y universal. (p.28) 
Cabe destacar, que las Tecnologías de Información y Comunicaciones (TIC) corresponden a aquellas tecnologías que permiten manejar información, tales como, recibimiento, almacenamiento y distribución, así como también facilitar diferentes formas de comunicación e interacción entre actores humanos y/o sistemas electrónicos.

Las empresas también pueden dar uso a las tecnologías de información y comunicación TIC como un método de enseñanza en las capacitaciones, inducciones y entrenamientos de los trabajadores, permitiéndole así que por vía internet, o dando algunos cursos por video conferencias se pueda reducir tiempos perdidos, y bajar costos que tengan que ver con salones, refrigerios entre otros.

La rentabilidad de una empresa respaldada por el marketing va a depender de las actividades a desarrollarse, considerándose como una herramienta que deben conocer los empresarios para la obtención de un mayor patrocinio de un producto determinado. Por su parte, Ortega (2001), se refiere al marketing como un:

Término de origen inglés que se empezó a utilizar en Estados Unidos entre 1906 y 1911, y que debido al amplio alcance $y$ naturaleza del mismo, no ha encontrado una palabra equivalente en otros idiomas, por lo que se ha introducido así en la mayoría de ellos (p.12).
Dentro de las nuevas estrategias de mercado que buscan posicionar a la compañía dentro de las mejores, han surgido estas basadas en el estudio del comportamiento humano desde la base psicológica que intenta predecir las necesidades y conductas de este, direccionando a la empresa a tener éxito en el mundo comercial. Partiendo de la idea de que los componentes racionales (producto y dinero) son fácilmente copiables por la competencia, disminuyendo la ventaja, se considera pertinente, como aseguran Robinette, Brand y Lenz, (2001) "la aplicación de componentes emocionales, como la equidad, experiencia y energía, pues son difícilmente reproducibles por el otro y le agregará valor a sus productos, tratando de ser únicos y originales en el mercado" (p.23).

En efecto, el marketing es una herramienta que ayuda al posicionamiento de un producto o una marca dentro de un mercado; cuando un cliente decide comprar lo hace por el producto que le sea más apetecible y el valor lo da principalmente la calidad de este. A la hora de ofertar, la empresa siempre debe estar centrado en lo que está haciendo, sin descuidar el entorno en el que se está desenvolviendo.

Fisher y Espejo (2006), señalan que la estrategia de mercadotecnia "comprende la selección y el análisis del mercado, es decir, la elección y el estudio del grupo de personas a las que se desea llegar, así como la creación y permanencia de la mezcla de mercadotecnia que las satisfaga" (p.26). 
Otro tema que se suma es el marketing digital. Hernández (2016), señala que el marketing digital, también conocido como marketing electrónico:

Engloba herramientas, técnicas y estrategias de marketing a través de los medios digitales (correo electrónico, televisión digital, radio digital, vídeo y videojuego digital, sitios web, comunidades virtuales, etc.) y utiliza las tecnologías de la información y la comunicación (TIC) para alcanzar los objetivos de marketing de las empresas. Entre las posibles acciones de marketing digital están el posicionamiento en buscadores, las campañas publicitarias en internet, los CRM para la gestión de clientes o las transacciones de comercio electrónico (p.15).

Las principales herramientas de marketing digital que dispone una empresa son:

- Web 2.0, Posicionamiento SEO y Analítica Web: un sitio web dotado de usabilidad y de un diseño orientado hacia al usuario, que disponga de un posicionamiento óptimo en buscadores mediante técnicas de SearchEngineOptimization (SEO) y de un sistema de métricas que permita analizar los resultados de tus visitas web, es la base para transformar un sitio web en un escaparate comercial.
- Marketing por correo electrónico de permiso: el e-mail marketing utilizado con sabiduría (estilo personal, información útil, periodicidad adecuada y listas de correos segmentadas) tiene un notable índice de respuesta. Esta herramienta que permite difundir información, generar branding, fidelizar clientes, entre otras funcionalidades, está enfocada a dos objetivos principales: retención y generación de clientes.

- Redes sociales: Las acciones de marketing 2.0 enfocadas a las redes sociales facilitan una mayor difusión y visibilidad de la empresa, pero es esencial ofrecer un valor útil a los seguidores. Si personalizas las redes sociales, tendrás mayor capacidad para aumentar tus prospectos y retener a tus clientes actuales. No olvides medir el retorno de la inversión (ROI) de las redes sociales de tu empresa.

- Publicidad en internet: Las herramientas de publicidad que nos ofrecen los buscadores para anunciarnos en sus medios de búsqueda o redes de contenidos, permiten rápidamente dar a conocer nuestros productos, servicios o marcas a través de la red. La optimización oportuna de las campañas en internet permite alcanzar un ROI más eficiente y reducir costos.

Los resultados sólo se producen cuando las herramientas se aplican con inteligencia, de acuerdo a un plan de marketing que esté 
alineado con los objetivos generales de la empresa.

Gálvez (2011) señala que las redes sociales como:

\begin{abstract}
Herramientas deben basarse en diversos elementos como la participación social y la inteligencia colaborativa, acotando que estas representan un enlace entre las organizaciones y sus públicos, de manera que si las empresas de equipamiento de cocina aplican las estrategias adecuadas de mercadeo en redes sociales se fortalecería este enlace hasta posicionarlas (p.54).
\end{abstract}

Estos métodos innovadores de la mercadotecnia guían a cualquier empresa en la formación de sus estrategias para posicionarse en cualquier mercado. En el caso de la Panadería y Pastelería Dorada, C.A., ubicada en el municipio Valencia estado Carabobo, la ayudaran a establecer su marca en un ámbito que ha ido creciendo velozmente, como lo son los comercios a través de las redes sociales.

El posicionamiento en el mercado de un producto o servicio es la manera en la que los consumidores definen un producto a partir de sus atributos importantes, es decir, el lugar que ocupa el producto en la mente de los clientes en relación de los productos de la competencia. Los consumidores están saturados con información sobre los productos y los servicios. No pueden reevaluar los productos cada vez que toman la decisión de comprar. Para simplificar la decisión de compra los consumidores organizan los productos en categorías; es decir, posicionan los productos, los servicios y las empresas dentro de un lugar en su mente.

El posicionamiento se puede definir como la imagen de un producto en relación con productos que compiten directamente con él y con respecto a otros productos vendidos por la misma compañía.

De esta manera, se puede decir que el posicionamiento en el mercado se fundamenta en el lugar que tiene o toma un producto en la mente de los consumidores, de las ofertas que como empresa se ofrece a los clientes de manera que esta sea la seleccionada como primera opción dentro de su clase con respecto a otros competidores.

\section{Resultados de la investigación de campo}

De los resultados obtenidos en la aplicación de los instrumentos de recolección de información, como fueron los dos cuestionarios suministrados a los empleados y clientes seleccionados al azar, cuya intención fundamental era diagnosticar la situación actual de mercadeo en la Panadería y Pastelería Dorada, C.A., se resumen los siguientes aspectos, los cuales fueron organizados para su análisis en una matriz DOFA. 
Factores internos:

\section{Debilidades:}

- Deficiencia en la distribución y exhibición de los productos que comercializa, para conocimiento de posibles clientes potenciales.

- No existe personal capacitado para el uso de medios sociales, como estrategia de mercadeo digital.

- Cartera de clientes que requiere ser ampliada.

- Poco aprovechamiento de la tecnología.

- Ausencia de un plan de marketing digital.

\section{Fortalezas:}

- El establecimiento comercial de la empresa está bien ubicada geográficamente.

- Poseen un personal que presta un buen servicio y soluciona las necesidades de los clientes, para desarrollar un buen proceso de ventas en conexión con la cultura organizacional.

- La empresa identifica tener una orientación hacia el cliente.

- Los productos comercializados son de buena calidad.

- Poseen un nivel de inventarios adecuados y Sus precios competitivos.

- Poseen recursos financieros para inversión

- Poseen equipos y conexiones necesarias para implementar estrategias de mercadeo para el posicionamiento de los productos.

Por su parte los clientes estuvieron de acuerdo:

- Variedad en los productos.

- De bueno a regular son los precios de los productos.

- El tiempo de entrega es el esperado.

- Las condiciones del establecimiento de la empresa son adecuadas.

- Es buena la rapidez utilizada por la empresa para resolver los problemas de los clientes.

Factores externos:

\section{Oportunidades:}

- Crisis económica ocasiona que las empresas busquen disminuir costos las estrategias de marketing.

- Posibilidad de importar marcas reconocidas y conocimiento del uso de medios y canales digitales por parte de los consumidores.

- Los clientes utilizan las redes como medio de obtener información para sus compras.

\section{Amenazas:}

- Variabilidad en la tasa de cambio al adquirir las divisas. 
- Alta inflación, lo que encarece los costos de adquisición y alta presencia de competidores.

Según la opinión de los encuestados, se tiene, deficiencia en las estrategias de marketing, no existe personal capacitado para el uso de medios sociales, como estrategia de mercadeo digital, la cartera de clientes que requiere ser ampliada, poco aprovechamiento de la tecnología.

De acuerdo a los resultados obtenidos en la presente investigación, se pudo observar que la misma, presenta en la actualidad un problema de identificación por parte del público consumidor, ya que la mayoría de los clientes se sorprenden al saber de la existencia de la misma y la variedad de productos con la que cuenta, esto es debido a que no se creó desde sus inicios un plan publicitario para su promoción $y$ conocimiento, la cual ha motivado a que la empresa no alcance las metas de productividad y posicionamiento planteadas. Esta empresa cuenta con una gama de productos y servicios que muchas veces es desconocido por un mercado de clientes que por desconocimiento no están cautivos.

Con los datos obtenidos de la investigación, se construye a continuación la Matriz estratégica DOFA, donde se cruzaran las Fortalezas y debilidades internas con las Oportunidades y Amenazas del medio ambiente externo, para plantear las estrategias adecuadas.

Cuadro 1. Estrategias de matriz DOFA

\section{FACTORES INTERNOS}

\begin{tabular}{|c|c|}
\hline Fortalezas & Debilidades \\
\hline $\begin{array}{l}\text { F.1. Buena ubicación geográfica } \\
\text { del establecimiento comercial. } \\
\text { F.2. Personal que presta un buen } \\
\text { servicio y soluciona las } \\
\text { necesidades de los clientes. } \\
\text { F.3. Orientación al cliente } \\
\text { F.4. Alta calidad de los productos } \\
\text { comercializados. } \\
\text { F.5. Nivel de inventarios y precios } \\
\text { competitivos. } \\
\text { F.6. Recursos financieros para } \\
\text { inversión. }\end{array}$ & $\begin{array}{l}\text { D.1. Deficiencia en la distribución y } \\
\text { exhibición de los productos } \\
\text { que comercializa. } \\
\text { D.2. No existe personal capacitado } \\
\text { para el uso de medios sociales. } \\
\text { D.3. Cartera de clientes que } \\
\text { requiere ser ampliada } \\
\text { D.4. Poco aprovechamiento de la } \\
\text { tecnología } \\
\text { D.5. Ausencia de un plan de } \\
\text { marketing digital. }\end{array}$ \\
\hline
\end{tabular}




\begin{tabular}{|c|c|c|}
\hline $\begin{array}{l}\text { FACTORES EXTERNOS } \\
\text { Lista de Oportunidades }\end{array}$ & ESTRATEGIAS (FO) & ESTRATEGIAS (DO) \\
\hline $\begin{array}{l}\text { O.1. Crisis económica } \\
\text { ocasiona que las } \\
\text { empresas busquen } \\
\text { disminuir costos las } \\
\text { estrategias de } \\
\text { marketing. } \\
\text { O.2. Posibilidad de } \\
\text { importar marcas } \\
\text { reconocidas. } \\
\text { O.3. Conocimiento del uso } \\
\text { de medios y canales } \\
\text { digitales por parte de } \\
\text { los consumidores. }\end{array}$ & $\begin{array}{l}\text { * Establecer contacto con el } \\
\text { cliente de manera más rápida } \\
\text { por la ubicación del } \\
\text { establecimiento comercial. } \\
\text { * Creación de página web, } \\
\text { donde se resalte los atributos } \\
\text { de los productos (calidad, } \\
\text { variedad) precios competitivos, } \\
\text { ubicación física de la empresa } \\
\text { y estrategias promocionales. } \\
\text { * Ampliar la presencia en nuevas } \\
\text { medios sociales, con la } \\
\text { finalidad de generar dialogo, } \\
\text { atención al cliente y tráfico } \\
\text { hacia la página web. }\end{array}$ & $\begin{array}{l}\text { * Mejorar la ubicación de los } \\
\text { productos para una mejor } \\
\text { exhibición de las marcas. } \\
\text { * Generar condiciones para lograr } \\
\text { el acercamiento hacia a los } \\
\text { clientes y conseguir la } \\
\text { comunicación empresa cliente } \\
\text { en ambos sentidos. } \\
\text { * Aprovechar los recursos } \\
\text { tecnológicos para poder } \\
\text { implementar las estrategias de } \\
\text { marketing digital. }\end{array}$ \\
\hline Lista de Amenazas & ESTRATEGIAS (FA) & ESTRATEGIAS (DA) \\
\hline $\begin{array}{l}\text { A.1. Variabilidad en la tasa } \\
\text { de cambio al adquirir } \\
\text { las divisas. } \\
\text { A.2. Alta inflación, lo que } \\
\text { encarece los costos } \\
\text { de adquisición. } \\
\text { A.3. Alta presencia de } \\
\text { competidores. }\end{array}$ & $\begin{array}{l}\text { * Establecer alianzas con } \\
\text { proveedores nacionales. } \\
\text { * Adecuar el nivel de inventario } \\
\text { en función de reponer } \\
\text { eficientemente los productos } \\
\text { más demandados. } \\
\text { * Establecer comparaciones de } \\
\text { los precios de la competencia. }\end{array}$ & $\begin{array}{l}\text { * Crear promociones especiales } \\
\text { para mejorar las ventas } \\
\text { * Generar confianza en los } \\
\text { productos que no sean de marcas } \\
\text { reconocidas. } \\
\text { * Contratar a personal competente } \\
\text { y calificado para implementar } \\
\text { estrategias de marketing digital. }\end{array}$ \\
\hline
\end{tabular}

Conforme a las necesidades evidenciadas, se propone a la empresa la implementación de estrategias de mercadeo para el posicionamiento de los productos, ya que este traerá a corto y mediano plazo a los clientes, proveedores directos y empleados, motivado por la inversión en publicidad que se implementaran, generando más ventas de los productos, reconociendo de la excelencia y calidad de los servicios de la empresa. 
Cuadro 2. Plan de Ejecución de la Propuesta

\begin{tabular}{ll}
\hline \multicolumn{1}{c}{ ESTRATEGIA } & \multicolumn{1}{c}{ ACTIVIDADES } \\
\hline Estrategias de & $\begin{array}{l}\text { Se propone dirigir las estrategias al público } \\
\text { Marketing Digital }\end{array}$ \\
& $\begin{array}{l}\text { enaneral en todos los productos de la donde además de ser } \\
\text { económicamente productivas, utilizan los } \\
\text { avances tecnológicos }\end{array}$
\end{tabular}

Estrategias de

Posicionamiento

\section{Estrategias de Marketing Mix}

Generar contenido diferenciadores respecto a la competencia (promociones, servicio y atributos de los productos).

* Establecer alianzas con otras empresas de asesoría, para la publicación de Banners alusivos a la Panadería y Pastelería Dorada, C.A.

\section{Producto:}

Adecuar el nivel de inventario en función de reponer eficientemente los productos más demandados.

* Generar confianza en los productos que no sean de marcas reconocidas.

* Mejorar la ubicación de los productos para una mejor exhibición de las marcas.

\section{Precio:}

- Establecer alianzas con proveedores nacionales que faciliten la adquisición de productos de diferentes marcas.

* Ofrecer descuentos por suscripción a la página Web.

\section{Plaza:}

Relacionar el establecimiento físico de la empresa con la información dada a conocer a través de la página web.

\section{Promoción:}

- Indicar una sección de preguntas frecuentes en la página web.

* Crear un programa de afiliación, que genere incentivo a los consumidores.

\section{Personas:}

Definir la persona encargada del manejo de la página web y los medios sociales (Community Managger).

\section{METAS}

Posicionamiento de los productos que ofrece la Panadería y Pastelería Dorada, C.A. ubicada en el municipio Los Guayos estado Carabobo.

Dar a conocer la Panadería y Pastelería Dorada, C.A.., los productos $\mathrm{y} / \mathrm{o}$ servicios a través de diferentes medios sociales más utilizados por los consumidores (Redes Sociales y pagina web)

Aumentar la capacidad informativa de los productos (especificaciones técnicas), modelos, marcas y servicios que ofrece la empresa.

Definir precios competitivos respecto a la competencia.

Establecer contacto con el cliente de manera más rápida por la ubicación del establecimiento comercial.

Crear promociones, descuentos $y / o$ concursos para mejorar las ventas y dar a conocer esta información a través de la página web y los medios sociales.

Establecer criterios para el uso de las plataformas creadas que permitan dar a conocer la empresa a un mayor número de consumidores. 


\section{Estrategias de Comunicación}

- Define cómo los visitantes son llevados al sitio web desde otros sitios web o a través de comunicaciones en otros canales.

* Traer visitantes a un sitio web desde otros sitios web (medios sociales), con el fin de establecer comunicación con las audiencias a través de medios digitales para lograr los objetivos de negocio.

* Atraer visitantes al sitio web o conseguir conocimiento de marca en sitios de terceros.

* Trasladar al sitio web desde otros medios no digitales con los segmentos objetivos a través de marketing directo en el establecimiento físico de la empresa, comunicando nuestra propuesta de valor online.

* Utilizar el canal online para conseguir ventas en canales offline.

- Comunicar promociones de primera compra.

* Optimización del diseño del sitio web, actualizando el contenido constantemente
Comunicarse con los segmentos a través de la publicidad interactiva, marketing en buscadores, patrocinios, acuerdos con terceros, email y marketing viral.

Fuente: Aponte M, Beiza Y, Duran E. (2019)

A continuación para dar continuidad a la propuesta de la presente investigación, se presentan algunas de las cuentas en medios sociales (Facebook, Instagram y Twitter) y pagina web.

Página de Facebook: En la página principal de esta red social se podría apreciar el perfil público identificado con el nombre de la Panadería y Pastelería Dorada, C.A. Este medio social, permitiría dar a conocer datos muy importantes como Información de la empresa, números de contactos, dirección, publicaciones de promociones y se podrían indicar enlaces a la otra red social y pagina web de la empresa creando de esta manera integración entre las mismas.

Página de Instagram: En este medio social, se podría dar a conocer información de la actividad que realiza la empresa y ubicación, además permitiría publicar imágenes acompañadas con mensajes escritos que genere interés por parte de los consumidores e informar sobre productos, novedades ofertas, ofertas y enlace de la página web de la empresa. 
Página Web: La página web brindará a la empresa, la oportunidad de generar contenido, adaptado a las necesidades de los consumidores, dar a conocer los productos que ofrece en forma detallada exponiendo características y atributos de los mismos, ofertas, información de contacto, suscripción a boletines de información y una sesión de registro que permitiría a la empresa obtener datos de contacto de los consumidores.

\section{CONCLUSIONES}

En la investigación que tuvo por objetivo general proponer el marketing digital como estrategia que permita el posicionamiento en el mercado de la Panadería y Pastelería Dorada, C.A., ubicada en el municipio Los Guayos estado Carabobo; se puede concluir que dicha empresa desarrolla una labor satisfactoria para sus consumidores pero aún falta implementar estrategias que aporten a la captación de nuevos clientes y posicionarse en el mercado a pesar de la amplia competencia a la que se enfrenta.

El conocimiento de las herramientas de comunicación digital por parte de los clientes es una ventaja en la actualidad ya que por tratarse de una población grande que hace uso de ella con frecuencia, se convierten en consumidores potenciales que pudieran fidelizarse a la empresa por medio de estrategias de mercadeo como lo es el marketing digital. Lo que permite concluir que los productos y servicios de la empresa, están dirigido a un extenso número de personas.
A partir de lo anterior, se puede hacer una serie de recomendaciones orientadas a la captación de nuevos clientes y posicionamiento a través de estrategias de marketing digital que aporten a la aplicación de un plan estratégico de mercado, considerando puntualmente las siguientes sugerencias:

- Difundir información constante de los productos que ofrece la empresa donde se especifiquen de manera clara la descripción, marca y precios de los productos.

- Tomar en cuenta la opinión de los clientes para la satisfacción de los mismos, proporcionando así la confianza que necesitan a la hora de adquirir un producto.

- Implementar estrategias de diseño mediante la utilización de medios digitales; las redes sociales, blogs y páginas web pueden ser canales de comunicación para la interacción cliente-empresa.

- Ejecutar y Desarrollar el plan de mercado propuesto a corto plazo tomando en cuenta que puede ser aplicable a otras empresas.

- Instruir al personal encargado de ofrecer la información referente a la empresa y a los productos, a través de realización de cursos, capacitando a los mismos para la atención de clientes.

- Determinar si es rentable la aplicación de la propuesta, a través de un estudio económico y de mercado. 
- Realizar la planificación estratégica adecuada para enfrentar el mercado potencial, la competencia, entre otros factores que rodee la empresa, con la finalidad de beneficiarse con la captación de nuevos clientes y mantener la satisfacción de los clientes actuales.

\section{REFERENCIAS}

Fisher, Luís y Espejo, José (2006). Mercadotecnia. Editorial: MCGRAWHILL. México

Gálvez, Ivis (2011). Introducción al Marketing en Internet: Marketing 2.0. España. IC Editorial

Gaynor, Betza. (1999). El Ordenador Invisible. España: Editorial Gedisa.

Gil, E. (2002). Identidad y Nuevas Tecnologías. Disponible en:

http:www.voc.edu/web/esplart/gil0902/htm.

Consulta : 14/01/2020

Hernández, Fermín (2016). Plan y Marketing

Digital: La Pareja Perfecta 2.0.

Hughes, Gaynor (2007). Mercadotecnia.

Planeación Estratégica. Estados Unidos.

Addison-Wesley

Koontz, Harold y Weihrich, Heinz (1998).

Administración. Una Perspectiva

Global. $11^{a}$ Edición. Editorial Mc GrawHill Interamericana. México
Kotler, Peter (2002). El Marketing Según Kotler. Cómo Crear, Ganar y Dominar Los Mercados. Paidós Empresa, Buenos Aires

Ortega, E. (2001). El mensaje y los fundamentos económicos del marketing. Editorial ESIC, Madrid

Peinado, Vivian y Prado, Roberto (2008). Propuesta de una guía general de acción para la aplicación del marketing digital en las pequeñas y medianas empresas (PYMES), en la ciudad de Cumaná, Estado Sucre. Trabajo Especial de Grado no publicado, Universidad de Oriente, Cumaná

Robinette, S., Brand, C. y Lenz, V. (2001). Marketing Emocional. Gestión, Barcelona

Valdés, Luis (2000). El Sistema Tecnológico en las Organizaciones y su Administración. Disponible en: http://www.tecnologiaycalidad galeo.com/tecnologia/1.htm

Vargas, Saúl (2015). Nuevas Formas de Publicidad y Mercadeo en la Era Digital: Una Mirada Exploratoria A Comunidades, Portales Interactivos y Advergames. Trabajo de grado para optar por el título de Comunicadora social con énfasis en Publicidad y Periodismo 